\title{
Crystalline growth under electric field: toward a new route to design domain modulated-structures. Case of $\mathrm{CaTiO}_{3}$
}

\author{
M. Pellen ${ }^{1}$, R. Saint-Martin ${ }^{1}$, R. Haumont ${ }^{1,2, \not}$ \\ ${ }^{1}$ Institut de Chimie Moléculaire et des Matériaux d’Orsay, Université Paris-Sud, Université Paris-Saclay, UMR CNRS 8182, \\ 91405 Orsay, France \\ ${ }^{2}$ Laboratoire Structure, Propriétés et Modélisation des Solides, CentraleSupelec, UMR CNRS 8580, Université Paris-Saclay, \\ 91190 Gif-sur-Yvette, France \\ қ contact : raphael.haumont@u-psud.fr
}

Keywords: A2.Floating zone technique; A2. Single crystal growth; B1. Perovskites; A1. Directional solidification

\section{Abstract}

A single crystal of $\mathrm{CaTiO}_{3}$ was prepared by the floating method (FZ) method in which a high external electric field $\left(\geq 3 \mathrm{kV} . \mathrm{cm}^{-1}\right)$ is applied. Such an electric field is a new powerful tool, in crystalline growth, able to create new chemical structures and original new materials with new physical properties. By varying crystal growth velocity and electric field strength, we show that we can control the macroscopic shape of $\mathrm{CaTiO}_{3} \mathrm{single}$ crystals, and we can alter the crystalline orientation of domains. Applying an in-situ electric field during crystalline growth is a new tool to design domain modulated-structures.

\section{Introduction}

Since ferroelectricity has been observed in the perovskite oxide $\mathrm{BaTiO}_{3}, \mathrm{ABO}_{3}$ perovskites have been the subject of many studies. These compounds are widely investigated due to the high potential for technological application of their diverse physical properties [1-4]. Many of the existing and future industrial applications of perovskites (especially ferroïcs) are based on the capability of the structure to respond to external forces (mechanical stress, electric field, magnetic field, etc.). The remarkable properties of these materials come not only from chemical competitions (i.e. chemical, polar, magnetic orders) but also the intrinsic macroscopic domain configuration. For example, the remarkable piezoelectric responses of morphotropic lead-based perovskites (e.g. $\mathrm{Pb}(\mathrm{ZrTi}) \mathrm{O}_{3}$ (called $\mathrm{PZT}$ ), $\mathrm{Pb}\left(\mathrm{Mg} / \mathrm{Nb} / \mathrm{Ti} \mathrm{O}_{3}\right.$ (called PMN-PT), etc.) can be explained by complex local polar (dis)orders, cationic competitions, and the mobility of ferro-domains under stress, temperature or external electric field [5]. Due to their remarkable electromechanical coupling factors, these compounds have opened a new way to approach real industrial solutions for energy harvesting. In recent years, parts of roads, parking areas and subway platforms (e.g. the JR line of Tokyo) have been used to harvest the energy coming from cars or pedestrians. In the same manner, flexible piezoelectric smartphones, self- 
powered implantable cardiac devices and electric generators based on wind vibrations have been developed and tested $[6,7]$.

From an industrial point of view, the work mainly consists of optimizing the shape (ceramic, powder, crystal, composite, etc.), and play of polarization (selecting and orienting piezo domains) of the material. This step is complex and requires selecting the piezoelectric resonance mode and heating or cooling the material under an electric field (piezoelectric domain engineering). Representative piezoelectric materials can be categorized into piezoceramics and piezopolymers. Piezoceramics (such PZT) have large electro-mechanical coupling constants and provide high energy conversion rates, but they are too brittle to use as a general shape energy transducer. On the other hand, piezopolymers (e.g. PolyVinyliDene Fluoride) have smaller electromechanical coupling constants compared to the piezoceramics but are very flexible. Recently, Karami, et al. [8] examined different configurations of three piezoelectrics (a single crystal PMN-PZT and two polycrystalline PZT ceramics) in a unimorph cantilevered beam to find the best design configuration for lightweight energy harvesting devices for low-power applications. They concluded that single-crystal energy harvesters produced superior power compared with polycrystalline devices. P. Rakbamrung, et al. [9] have shown that although a PMN-PT-based harvester harvested approximately twice the power of a PZT-based device when using a classical electrical interface, then using a nonlinear approach for enhancing the conversion capabilities of piezoelectric elements dramatically reduced the difference between the micro-generators considered for energy harvesting. Moreover, even if Pb-based perovskites are very attractive, the REACH standard asks industry to replace lead-based materials [10]. As a result, both scientists and industry must focus their research on new materials (without $\mathrm{Pb}$ ) and new processes.

We recently developed a new apparatus to apply a high electric field during the growth of a single crystal. Recent works reported the role of the electric field on kinetic and thermodynamic processes during crystalline growth [11]. In the selection, orientation and distribution of piezoelectric domains, Hicher and al. showed that an electric field acts like an external force [12]. The electric field can act on thermodynamic equilibrium and on a solid/liquid interface [13]. Its application during growth modifies nucleation [14], the partition coefficient of species [15] and the possible modification phases diagrams of a material [11]. Indeed ions within a difference of electric potentials see their energy changing, which implies a new thermodynamic equilibrium [16]. Thus, applying an intense electric field during compound growth is a new parameter to affect the direction of polarization and possibly to amplify the value of polarization by exacerbating ionic displacements.

In light of these papers, we are confident that this new crystalline growth method will open new opportunities to increase, control and modulate physical properties to develop more effective energy harvesting solutions. Additionally, to study the impact of domain structures, we have focalized our attention on the crystalline growth of ferroelectric $\mathrm{CaTiO}_{3}$. Even if $\mathrm{CaTlO}_{3}$ is a nonpolar material, its remarkable intrinsic ferroelastic domain state makes it a good candidate to test the impact of the electric field during the crystalline growth. Herein we report experiments using different values of crystal growth velocity VG and electric field strength $\vee$ parameters. Our investigations show that we can control the macroscopic shape of a single $\mathrm{CaTiO}_{3}$ crystal and play on domain distribution. 
$\mathrm{CaTiO}_{3}$ is well studied and its ferroelastic properties are well-known. The crystals grow easily at atmospheric pressure using the floating zone method. Crystalline growth involves competition between surface energy, bulk growth, and preferential orientation. Applying an electric field during the growth of such material can drastically modify ferroelastic domains. Yokota et al. [17] proved the existence of polar domain walls. The growth of this compound under an electric field should have an impact on these walls. $\mathrm{CaTiO}_{3}$ is orthorhombic with a spatial Pbnm group below $1380 \mathrm{~K}$ with the lattice parameters $\mathrm{a}=0.5524 \mathrm{~nm}, \mathrm{~b}=0.764 \mathrm{~nm}$ and $\mathrm{c}=0.538$ $\mathrm{nm}$. Above $1580 \mathrm{~K}$, it becomes cubic with a $\mathrm{Pm}-3 \mathrm{~m}$ spatial group. $\mathrm{CaTiO}_{3}$ is widely used in ceramic materials for electronic applications. It is a key component of Synroc, a synthetic rock form used to immobilize nuclear waste $[18,19]$. It has great potential due to its phase transition at high temperature and pressure and its dielectric constant at high temperature [20].

Single $\mathrm{CaTiO}_{3}$ crystals were grown using the floating zone (FZ) method to which we added a high external electric field (up to $3 \mathrm{kV} \mathrm{cm}^{-1}$ ). These studies indicated that the direction of macroscopic crystal growth results from a compromise between the direction of the imposed electric force $\left(F_{\text {elec }}\right)$ and the direction of the driving force ( $\left.F_{G r o w t h}\right)$. The driving force is equal to the thermal gradient in the furnace [21] (according to $z$ in the melting furnace of the zone called "vertical").

We want to analyse and observe the impact of an electric field on single crystal, specifically on its ferroelastic domains and the possibility of controlling them. We experimented to determine how an electric field affects the macroscopic properties of $\mathrm{CaTiO}_{3}$. The use of an electric field seems to substantially change the growth conditions. The aim of this work is to study and understand the role of electric fields on the kinetic and thermodynamic processes during growth and orientation.

\section{Experimental methods}

The polycrystalline $\mathrm{CaTiO}_{3}$ feed road was prepared by a conventional solid-state reaction. Powders of $\mathrm{CaCO}_{3}$ and $\mathrm{TiO}_{2}$ with a purity of $99.99 \%$ were mixed together in stoichiometric proportions for 20 min in an agate mortar. The starting powder was placed at $1580 \mathrm{~K}$ for $24 \mathrm{~h}$ in air in an alumina crucible. The powder was crushed and placed again $1580 \mathrm{~K}$ for $24 \mathrm{~h}$ in air for a second thermal treatment. After that, the powder was shaped into a rod in a latex tube, with a diameter of $6 \mathrm{~mm}$ and a length of $80 \mathrm{~mm}$, isostatically pressed (2000 bars) and sintered in air at $1550 \mathrm{~K}$ for $24 \mathrm{~h}$. After each treatment, phase purity of the sample was evaluated with X-ray diffraction. The pure $\mathrm{CaTiO}_{3}$ perovskite orthorhombic phase was observed.

The synthesis of a high-quality single crystal was performed under air with an optical floating-zone furnace (NEC SC-N15HD). The heat source of the FZ furnace is 2 halogens lamps (1500 W each). The temperature during the experiments can be controlled by the power of the lamps but cannot be measured directly. An electric field was applied using a high voltage generator (Spellman SL10, $10 \mathrm{~mW}$ ) connected to an experimental device (Figure 1). A camera was used to monitor growth. We can reach a high voltage (above 10 kV) with weak intensity (in the micrometre range). Electrodes were placed obliquely on both sides of the melting zone to apply an electric field through the liquid-solid interface. The distance between electrodes was about $3 \mathrm{~cm}$ with an angle of $40^{\circ}$. The experiments were conducted with about $78 \%$ lamp power. We have 
already reported simulation of electric field (comsol software) combined with experimental data that proved that electric field crosses the solid-liquid interface [12].

The first results show that $\mathrm{CaTiO}_{3}$ is very sensitive to the electric field. We will see in the next section that phenomena occur when several parameters during the growth are modified. Growth under an electric field shows competition between an electric force $\left(F_{\text {elec }}\right)$ and the driving force of crystallisation ( $\left.F_{\text {growth }}\right)$ (Figure 1). Single crystal morphology changed and these observed effects were compared and analysed.

To study the electric field effect, we conducted crystal growth without an electric field for few centimetres and then we applied it. We varied two parameters during the monocrystal growth, the pulling rate or growth velocity $\left(\mathrm{V}_{\mathrm{G}}\right)$ from 1 to $10 \mathrm{~mm} / \mathrm{h}$ and the electric field strength (V) from 2 to $9 \mathrm{kV}$. Otherwise, all experiments were conducted without crystal rotation and in air. Rotation of the upper rod was about $8 \mathrm{rpm}$. The observed effects on all the crystals obtained were then compared.

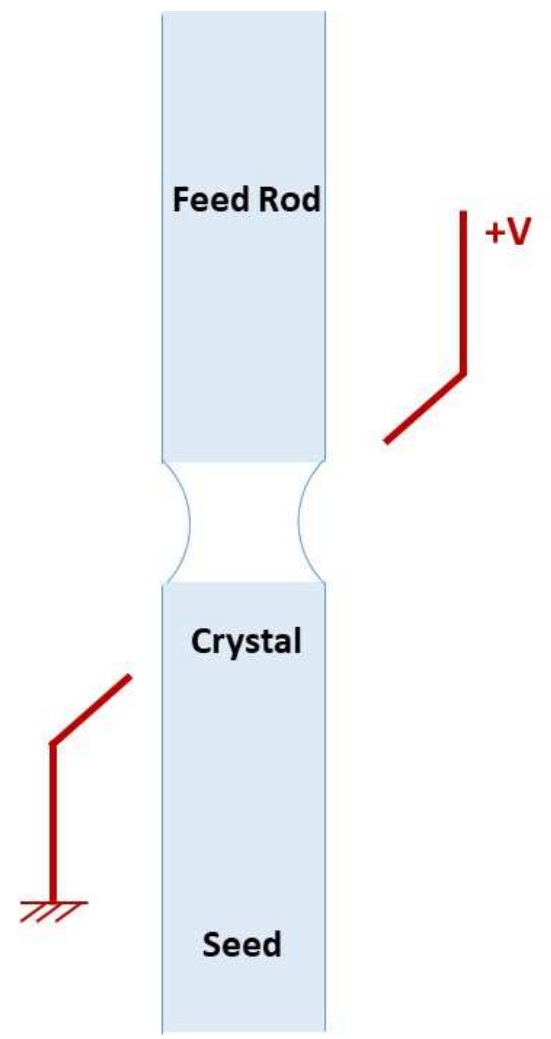

Figure 1: Experimental device with an electrode applying an electric field 


\section{Results}

\section{a) Electric field effect}

The first observation of the effects of applying an electric field during crystal growth is an inflection of the crystal (Figure 2a). We chose to apply a constant growth velocity, $\mathrm{v}_{\mathrm{G}}=3 \mathrm{~mm} / \mathrm{h}$ and began the crystal growth without applying an electric field for the first $2 \mathrm{~cm}$ of growth. No change was noticed in the solid-liquid interface or in the crystal shape. Afterwards, we applied an external electric field with an increasing voltage until reaching about $7 \mathrm{kV}$. We continued the growth until producing a crystal with a length of about $5 \mathrm{~cm}$ and a diameter of about $0.5 \mathrm{~cm}$ (Figure 2a). Crystal growth did not disturb the monocrystalline state previously attained even though the solid-liquid equilibrium changed. We chose to cut three slices of about 2-3 mm from our sample (Figure 2b). Two slices were cut from the plane perpendicular to the growth direction, the first where the sample grew without an electric field and the second with an electric field. The last one was also cut in electric field part but perpendicular to the crystal growth direction. All the slices were optically polished to analyse their orientation. The orientation of the crystal was measured by the Laue method. The [-10-1]pc axis in pseudo-cubic coordinates (pc) is clearly identified for all the single crystal slices for preferential axis growth.

However, as we can see in Figure 2, an inflection of about $\theta=8^{\circ}$ occurs after the application of the electric field. The inset pictures of Figure $2 b$ show that the $[-10-1] p c$ axis has also been deflected by an angle of about $8^{\circ}$. However, in the Laue pictures, even if the growth axis tilted during application of the electric field, the crystal orientation remains unchanged. 


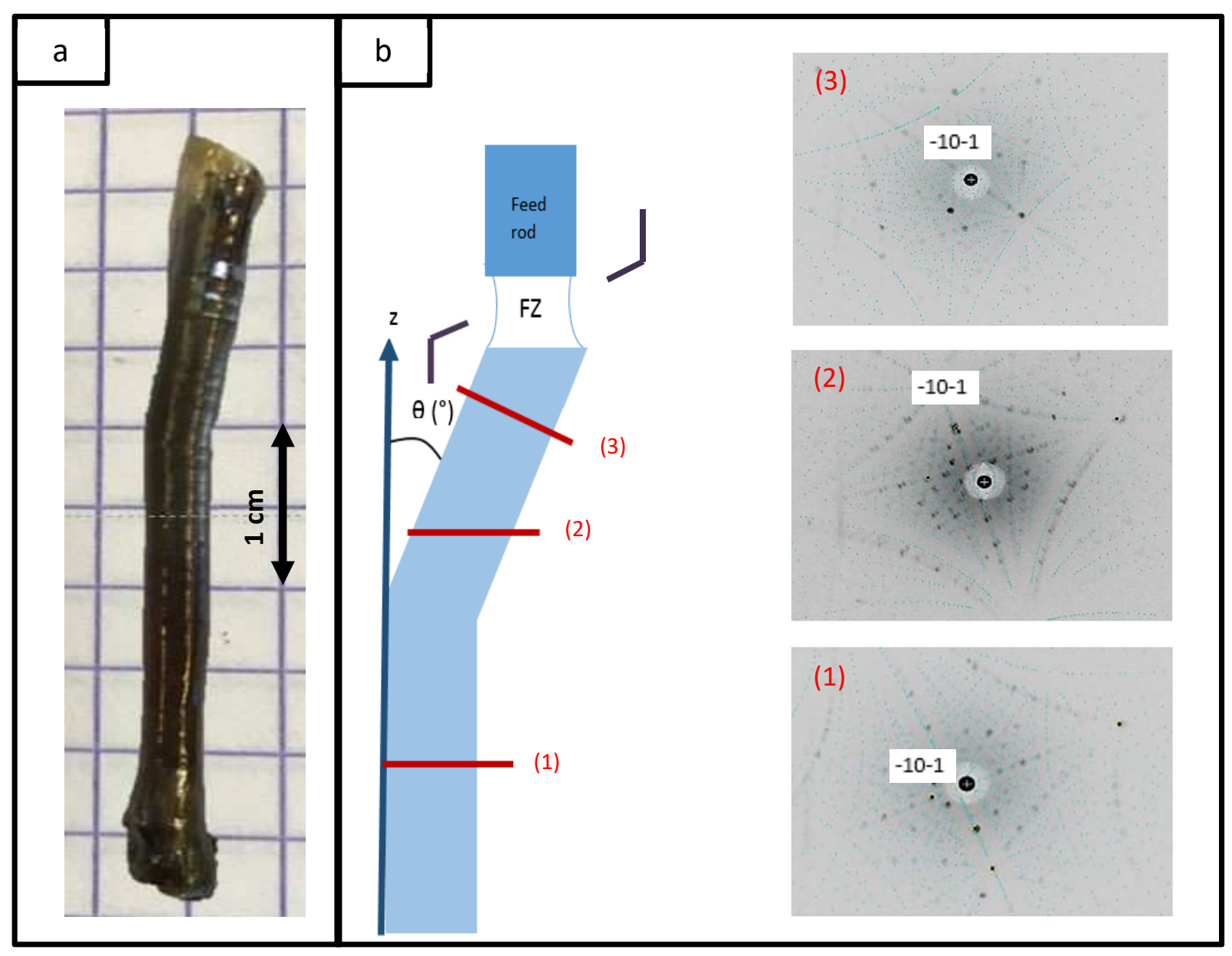

Figure 1: a. photograph of a $\mathrm{CaTiO}_{3} \mathrm{crystal}$, b. different cut of the crystal and orientation results by Laue method 


\section{b) Electric field $(V)$ and crystal growth velocity $\left(v_{G}\right)$ effects}

To evaluate the effect of an electric field on $\mathrm{CaTiO}_{3}$ single crystals, we performed a set of experiments. Figure 3 shows the evolution of the crystal inflection as a function of the electric field strength with different growth velocities. Five different crystal growths were conducted by modifying two parameters: growth velocity $\left(v_{G}\right)$ and electric field intensity $(V)$. The other parameters remained unchanged.

These results show that the $\mathrm{CaTiO}_{3}$ liquid zone is very sensitive to both the electric field and the growth velocity. Three regions are highlighted.

In Region I, below about $3 \mathrm{kV}$, almost no inflection of the crystals has been noticed. From a macroscopic point of view, the electric field has no impact on crystal growth. Below this threshold, the field is weak and negligible against the 'natural' crystalline growth force. In Region II, when the electric force is greater than $3 \mathrm{kV}$, the crystals progressively inflect with $\mathrm{V}$. This inflection can reach $\theta=10^{\circ}$ for a voltage up to $9 \mathrm{kV}$. The angle of deviation is proportional to the intensity of the electric field force. In Region III, a threshold angle is attained; saturation is obtained. A further increase of the electric field force didn't change crystal shape and inflection when this equilibrium is reached. The angle seems saturates around $10^{\circ}$. It seems that crystals try to line up along the field lines; however, a restoring force (vertical symmetry of the melting furnace) prevents a stronger inflection.

Furthermore, growth velocity modifies the range of these different regions, except for Region I. Below 3 $\mathrm{kV}$ (and for all growth velocities tested), the electric force is not strong enough to modify the crystal interface. The lower the $v_{G}$, the faster the threshold angle is reached. With a low growth velocity $\left(v_{G}=1 \mathrm{~mm} / \mathrm{h}\right.$ for example) the threshold is about $5 \mathrm{kV}$ while for a high growth velocity (such as $\mathrm{VG}_{\mathrm{G}}=10 \mathrm{~mm} / \mathrm{h}$ ) the threshold is about $7 \mathrm{kV}$. This parameter influenced the growth interface and these results indicate competition between the driving force of crystallization and the electric force. For low velocity, the matter 'has time' to adapt to the electric force. Therefore, the maximum deviation is reached at a lower voltage. In contrast, a high crystalline growth velocity induces a high vertical crystalline force on the interface, which can easily counterbalance the electric force. Thus, the macroscopic deviation of the crystal is observed only if $\mathrm{V}$ is important. Consequently, with increasing growth velocity, the boundary between Region II and III is shifted towards higher voltage. 


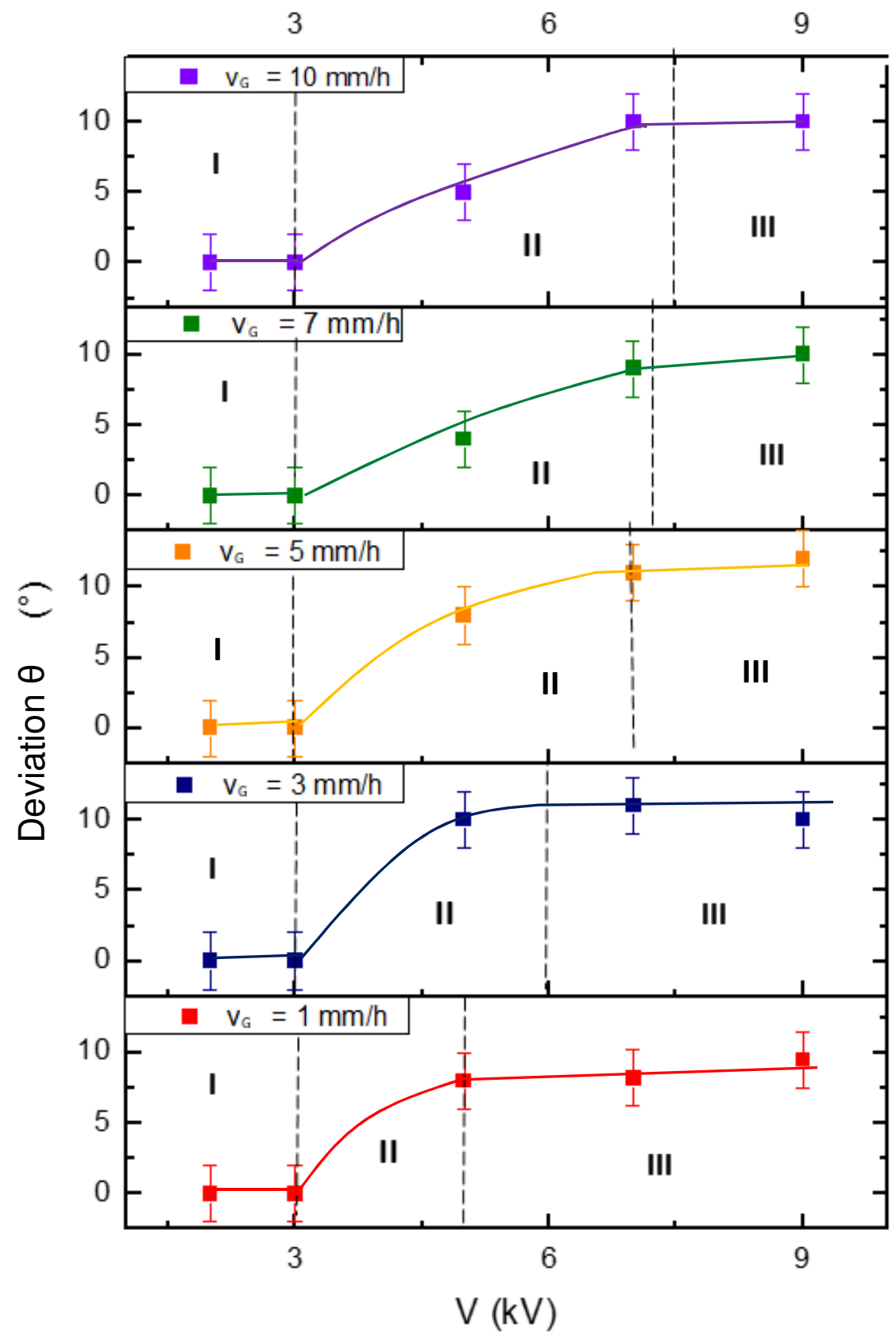

Figure 3: Evolution of crystal interface with electric potential $(V)$ and growth velocity $\left(v_{G}\right)$ 


\section{c) Domain Modulation and Design}

In order to prove the competition between the driving force of crystallization and the electric force, we performed experiments with an alternation $(0 /+V)$ of the electric potential applied during growth (see Figure 4). This alternation did not disturb the previously attained monocrystalline state even though the solid-liquid equilibrium was changed. However, the growth axis tilts during the application of the electric field without inducing a change of orientation. These orientations have been checked with the Laue method. This growth was performed at a $10 \mathrm{~mm} / \mathrm{h}$ velocity. The intensity of the electric field was increased by $3 \mathrm{kV}$ every 5 min until it reached a high field (here $9 \mathrm{kV})$. With a high growth velocity $(10 \mathrm{~mm} / \mathrm{h})$, crystal inflection is observed after 30 min of growth.

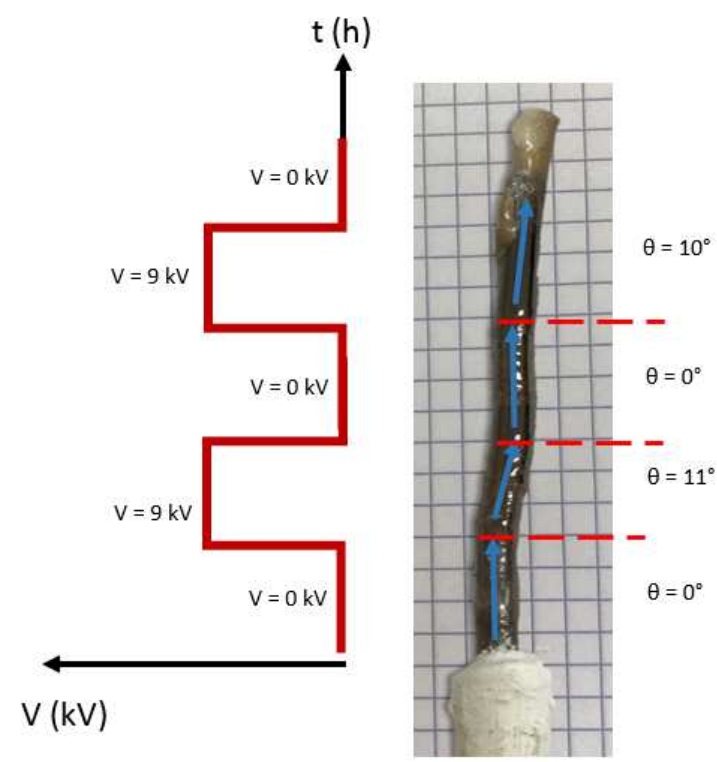

Figure 2: Modification of the crystal interface with an alternating electric field over the time $t$

The growth began without an electric field for $2 \mathrm{~cm}$, an electric field $(\approx 9 \mathrm{kV})$ was then applied for $1,5 \mathrm{~cm}$. As expected, the crystal tilted with an angle of $\theta \approx 11^{\circ}$. The generator was turned off and the growth continued for $2 \mathrm{~cm}$ more. Without the electric field's influence, the crystal grew following the natural driving force. Therefore, the phenomenon is reversible. When the generator is switched off, crystal growth follows the natural growth direction. 


\section{Conclusion}

By using the floating zone technique with the application of an electric field during growth, we demonstrated that different growth conditions provide different crystal orientations. This study reveals the existence of two threshold field/angles, one at about $3 \mathrm{kV}$ and another one depending on growth velocity ( $\left.\mathrm{V}_{\mathrm{G}}\right)$. Between these two values, crystal orientation tilted following the electric force until reaching about $10^{\circ}$. At field strengths less than $3 \mathrm{kV}$, the crystal didn't undergo an electric field effect, since no change of the spontaneous orientation has been noticed. However, above this value, the electric field is a new parameter for crystal growth, which competes with the growth velocity $\left(\mathrm{v}_{\mathrm{G}}\right)$. It is a new tool for domain modulation. Our investigations highlighted that according to the value of the couple $\left(\mathrm{V}_{\mathrm{G}}, \mathrm{V}\right)$, we can control the angle of deviation from the vertical direction of the crystal orientation. Many efforts (experimental and thermodynamic approaches) are needed to establish an equation which can represent the balance between the electric field strength and the growth velocity. A better understanding of the chemical composition of the liquid is required to predict the nature of the ions, their mobility under the field to better estimate the conductivity and even screening effect (which can explain existence of the electric field threshold). Moreover, we can expect that the driving force is dependent of the velocity, but also the geometry of the furnace, the thermal gradient, rotation speed of the feed rod etc....

Nevertheless, this by applying an alternating or increasing electric field we could interact with ferroelastic domains and modify them. It opens a new route to write data, control polarization during the growth of material and playing on the domain distribution of ferro-electric/elastic materials.

\section{References}

[1] A. Kojima, K. Teshima, Y. Shirai, T. Miyasaka. (2009). Organometal halide perovskites as visiblelight sensitizers for photovoltaic cells. J. Am. Chem. Soc., 131(17), pp. 6050-6051.

[2] L.G.G. Tejuca, L.GG. Tejuca, J. Fierro (2000). Properties and applications of perovskite-type oxides. CRC Press.

[3] A.Millis. (1998). Lattice effects in magnetoresistive manganese perovskites. Nature, 392, pp. 147150.

[4] R.Mitchell. (2002). Perovskites: modern and ancient. 3, Thunderbay, Ontario : Almaz Press.

[5] T. Tsurumi, T.Tsurumi, Y.Kumano, N.Ohashi, T.Takenaka and O.Fukunaga. (1997). 90 Domain reorientation and electric-field-induced strain of tetragonal lead zirconate titanate ceramics. Jpn. J. App. Phys., 36(9B), pp. 5970-5975.

[6] J.Sirohi and R.Mahadik. (2011). Piezoelectric wind energy harvester for low-power sensors. J. Intell. Mater. Syst. and Struct., 22(18), pp. 2215-2228. 
[7] G-T.Hwang, H.Park , J-H.Lee , S.Oh , K-I.Park , M.Byun , H.Park , G.Ahn , C.K.Jeong, K.No, H.Kwon , S-GLee, B.Joung, and K.J.Lee. (2014). Self-powered cardiac pacemaker enabled by flexible single crystalline PMN-PT piezoelectric energy harvester. Adv. Mater., 26(28), pp. 4880-4887.

[8] M.A.Karami, O.Bilgen, D.J.Inman, and M.Friswell. (2011). Experimental and analytical parametric study of single-crystal unimorph beams for vibration energy harvesting. IEEE Trans. Ultrason., Ferroelectr. Freq. Control, 58(7), pp. 1508-1520.

[9] P. Rakbamrung, M.Lallart, D.Guyomar, N.Muensit, C.Thanachayanont, C.Lucat, B.Guiffard, L.Petit, P.Sukwisut (2010). Performance comparison of PZT and PMN-PT piezoceramics for vibration energy harvesting using standard or nonlinear approach. Sens. Actuators A: Phys., 163(2), pp. 493-500.

[10] Commission, E. (2006, December 18). Environment. Récupéré sur http://ec.europa.eu/environment/chemicals/reach/legislation_en.htm

[11] S.Uda, X.Huang, S.Koh (2005). Transformation of the incongruent-melting state to the congruent-melting state via an external electric field for the growth of langasite. J. Cryst. Growth, 281(2-4), pp. 481-491.

[12] P. Hicher, R. Haumont, R. Saint-Martin, X. Mininger, P. Berthet, A. Revcolevschi. (2014). Experimental evidence that a high electric field acts as an efficient external parameter during crystalline growth of bulk oxide. J. Cryst. Growth, 409(1)pp. 23-26.

[13] S.Uda, X.Huang, S-Q.Wang. (2005). The effect of an external electric field on the growth of incongruent-melting material. J. Cryst. Growth, 275(1-2), pp. 1513-1519.

[14] X.Quan, G.Chen, P.Cheng. (2013). A thermodynamic analysis for heterogeneous boiling nucleation under an external electric field. Int. J. Heat Mass Transf., 65, pp. 308-313.

[15] S.Uda, W.A.Tiller. (1992). The influence of an interface electric field on the distribution coefficient of chromium in LiNbO3. J. Cryst. Growth, 121(1-2), pp. 93-110.

[16] R.Simura, K.Nakamura, S.Uda. (2008). Change of melting temperature of non-doped and Mgdoped lithium niobate under an external electric field. J. Cryst. Growth, 310(16), pp. 38733877.

[17] H. Yokota, H. Usami, R. Haumont, P. Hicher, J, Kaneshiro, E. K. H. Salje, Y. Uesu. (2014). Direct evidence of polar nature of ferroelastic twin boundaries in $\mathrm{CaTiO}$ obtained by second harmonic generation microscope. Phys. Rev, 89, pp. 144109.

[18] K Ueda, H Yanagi, H Hosono and H Kawazoe (1999). Study on electronic structure of $\mathrm{CaTiO}_{3}$ by spectroscopic measurements and energy band calculations. J. Phys.: Condens. Matter, 11(17), pp. 3535-3545.

[19] S. Saha, T. P. Sinha and A. Mookerjee. (2000). First principles study of electronic structure and optical properties of CaTiO3. Eur. Phys. J. B, 18(2), pp. 207-214.

[20] X.Wu, S.Qin, Z.Y.Wu, Y.H.Dong, J.Liu and X.D.Li. (2004). Study of $\mathrm{CaTiO}_{3}$ structure under high pressure, Acta Phys. Sin., 53(6), pp. 1967-1971.

[21] K. Kakimoto. (2013). Development of crystal growth technique of silicon by the Czochralski Method. Acta Phys. Pol. A, 124(2), pp. 227-230. 
Acknowledgments

Authors would thanks French ANR, JCJC program "RECIPE 2015-2018". Energy harvesting: Innovation in crystal growth of piezoelectric materials 